\title{
Inhibición y actualización en comprensión de textos: una revisión*
}

\section{Inhibition and Updating in Text Comprehension: a Review}

Recibido: 13 de abril de 2015| Aceptado: 28 de octubre de 2015

\author{
LUIS ÁNGEL ROLDÁN** \\ Universidad Nacional de La Plata, Argentina
}

doi: 10.11144/Javeriana,upsy15-2.iact

Para citar este artículo: Roldán, L.A. (2016). Inhibición y actualización en comprensión de textos: una revisión de investigaciones. Universitas Psychologica, 15(2) 87-96. http://dx.doi.org/10.11144/ Javeriana,upsy15-2.iact

* Articulo de revisión. Este trabajo específicamente no tuvo ninguna financiación.

** Profesor en Psicología, Facultad de Psicología de la Universidad Nacional de La Plata. Becario del Consejo Interuniversitario Nacional. Correo electrónico: angelroldan1990@gmail.com
RESUMEN

El presente trabajo describe un conjunto de investigaciones en las que se indagan las relaciones entre funciones ejecutivas y comprensión de textos. Se revisan específicamente de qué forma se asocian los procesos de actualización e inhibición con la construcción de un significado global del texto por parte del lector en un contexto determinado. Se seleccionaron investigaciones a través de las base de datos Redalyc, Scielo, Pepsic y Dialnet, bajos los descriptores "funciones ejecutivas" y "comprensión lectora". El corpus de investigaciones revisado no afirma en todos los casos que la inhibición sea discriminante de la comprensión exitosa. La actualización, en cambio, parece ser un buen predictor de la comprensión lectora en todos los trabajos revisados. Se puntualiza que los instrumentos diversos con que se han indagado ambas funciones ejecutivas limitan la comparación de resultados. Se propone para futuros estudios, realizar evaluaciones con similares instrumentos que permitan arribar a resultados comparables que sean la base para la evaluación y la intervención en comprensión de textos. Palabras clave

comprensión textual; funciones ejecutivas; actualización; inhibición

\section{A B S T R A C T}

The present work describes a group of investigations inquiring into the relationships between executive functions and text comprehension. Specifically, it is reviewed the way in which updating and inhibition processes are associated with the construction of global meaning in text by a reader in a certain context. The investigations were selected through Redalyc, Scielo, Pepsic and Dialnet databases, under the descriptors executive functions and reading comprehension. The reviewed investigations does not assert in all cases that inhibition is discriminant for succesful comprehension. Updating, however, seems to be a good predictor for reading comprehension in all of the surveyed works. It is stressed that the diverse instruments with which both executive functions have been analysed limit result comparison. For future studies, evaluations with similar instruments are proposed, so as to allow the obtaining of comparable results, which can in turn serve as a basis for evaluation and intervention in text comprehension.

Keywords

text comprehension; executive functions; working memory 


\section{Introducción}

La comprensión lectora es una habilidad de suma importancia para la inserción social-laboral de los individuos en un mundo cambiante y complejo. La comprensión textual, como fin último del aprendizaje de la lectura, es una temática que se encuentra actualmente en la agenda educativa. Esta preocupación por dicha herramienta cultural se evidencia en la inclusión de la misma en los programas de evaluación educativa a nivel nacional e internacional (Zabaleta, 2009) y en el desarrollo de pruebas específicas para evaluar el aprendizaje del lenguaje escrito, en nuestro país como en otros de habla hispana (Piacente, 2009, 2012)

Se entiende a la comprensión textual como la construcción de un significado global a partir del texto en un contexto determinado. Dicha habilidad multicomponencial involucra habilidades cognitivas y lingüísticas complejas (Abusamra, Ferreres, Raiter, De Beni, \& Cornoldi, 2010; Abusamra et al., 2011). Recientemente un conjunto de investigaciones se han preocupado por determinar cómo habilidades de control cognitivo o funcionamiento ejecutivo se involucra en el rendimiento académico en general y en el proceso de comprensión de textos en particular (Stelzer \& Cervigni, 2011; De Beni, Palladino, Pazzaglia, \& Cornoldi, 1998; Carreti, Cornoldi, De Beni, \& Romanó, 2005).

En el presente escrito se realiza una revisión de investigaciones que trabajan las relaciones entre las funciones ejecutivas de actualización e inhibición con la comprensión lectora. Se trata de una revisión sistemática en las bases de datos Redalyc, Scielo, Pepsic y Dialnet, bajos los descriptores: "funciones ejecutivas" y "comprensión lectora”. El objetivo del trabajo fue describir el modo en que los mecanismos ejecutivos mencionados se asocian o no a la comprensión lectora.

\section{Las funciones ejecutivas de inhibición y actualización}

La inhibición se refiere a un mecanismo cognitivo que se activa con el fin de suprimir información distractora que se presenta como competencia de la información relevante para los objetivos del sujeto (Cartoceti, 2012). La actualización, en cambio, se refiere a la modificación de la información contenida en la memoria a partir de nueva información que entra al sistema de procesamiento (Carreti et al., 2005).

Algunas perspectivas, postulan que la inhibición y la actualización forman parte de las funciones ejecutivas (Miyake, Friedman, Emerson, Witzki, \& Howerter, 2000), o más específicamente se incluyen dentro de los procesos ejecutivos de la memoria de trabajo (García Madruga, Gutiérrez Martínez, \& Vila Chaves, 2012).

Las funciones ejecutivas se refieren a un conjunto de mecanismos implicados en la optimización de los procesos cognitivos orientados a la resolución de problemas complejos (Tirapu-Ustárroz, MuñozCéspedes, \& Pelegrín-Valero, 2002).

El término "funciones ejecutivas" fue acuñado por la neuropsicóloga Muriel Deutsch Lezak, quien las describe como aquellas "capacidades mentales necesarias para formular metas, planificar la manera de lograrlas y llevar adelante ese plan de manera eficaz" (Lezak, 1982, citado por Musso, 2009 , p. 108).

En la revisión de literatura encontramos diferentes modelos teóricos que intentan dar cuenta del procesamiento ejecutivo. Existen modelos que piensan en las FE de manera unitaria, como un constructo único con sub-procesos asociados, otro grupo de enfoques conceptualiza a las FE como un conjunto de sub-procesos independientes entre sí, y finalmente una tercera orientación postula que las FE no son un constructo unitario, pero los componentes que las forman estarían asociados parcialmente (Musso, 2009; Tirapu-Ustárroz, García-Molina, Luna-Lario, Roig-Rovira, \& PelegrínValero, 2008a, 2008b).

Para este escrito son relevantes los planteamientos de las funciones ejecutivas de constructo único de Baddeley con el concepto de memoria de trabajo, y el modelo factorial de Miyake. (Tirapu-Ustárroz et al., 2008b).

El modelo factorial de Miyake et al. (2000) describe tres componentes ejecutivos diferenciables aunque no independientes, que contribuyen de manera diferencial al rendimiento en tareas de tipo ejecutivo. Los tres componentes son: 
1. Actualización: implica la monitorización, la manipulación y la actualización de información en línea en la memoria de trabajo.

2. Inhibición: consiste en la capacidad para inhibir de forma deliberada o controlada la producción de respuestas predominantes automáticas cuando la situación lo requiere.

3. Alternancia: capacidad de cambiar de manera flexible entre distintas operaciones mentales o esquemas, esta capacidad también se define como flexibilidad en la literatura consultada.

Por otro lado, Baddeley (2007 citado en García Madruga et al., 2012) en su conceptualización de la memoria operativa, acuñó un modo de funcionamiento ejecutivo en el procesamiento de la información del humano. Según este autor la memoria operativa o memoria de trabajo, se concibe como un sistema encargado de mantener y manipular la información que se necesita para realizar tareas cognitivas complejas. Según esta perspectiva, la memoria operativa estaría conformada por cuatro componentes: el bucle fonológico, la agenda viso-espacial, el retén episódico y el ejecutivo central.

El bucle fonológico y la agenda viso-espacial operan como un sistema de almacenamiento transitorio del material visual y auditivo. El retén episódico, sería el encargado de la conexión entre la memoria operativa y la memoria a largo plazo. Finalmente el ejecutivo central se encargaría de la coordinación de los componentes anteriores. Este último es el encargado de llevar a cabo los procesos ejecutivos, enfocando la atención, cambiando la atención y activando y actualizando representaciones de la memoria a largo plazo, inhibiendo procesos automáticos y descartando información irrelevante (García Madruga et al., 2012)

\section{Desempeño académico, comprensión de textos y funciones ejecutivas}

Existen variadas investigaciones que asocian el funcionamiento ejecutivo con el rendimiento académico en diversos dominios: matemáticas, ciencias, lectura y escritura, comprensión de textos, etc. (Clair-Thompson \& Gathercole, 2006; Stelzer \& Cervigni, 2011).

El funcionamiento ejecutivo también ha sido asociado con la comprensión lectora. Cuando hablamos de comprensión lectora nos referimos a una capacidad cognitiva compleja que implica construir una representación mental coherente del contenido de un texto. Para ello es necesario sostener, procesar y almacenar la información que proviene de este e integrarla con los conocimientos previos que ya posee el lector (conocimientos lingüísticos, conocimientos generales del mundo y conocimientos específicos de dominio) (Abusamra, Casajús, et al., 2011; Abusamra Ferreres, et al., 2010; De Beni, Cornoldi, Carretti, \& Meneghetti, 2003)

Los mecanismos de inhibición y actualización han sido indagados específicamente en relación con este constructo (Carreti et al., 2005; Carreti, Borella, Cornoldi, \& De Beni, 2009). Pero las investigaciones de referencia a este respecto han sido llevadas a cabo en su mayoría por fuera de Iberoamérica. Resulta de importancia entonces, poder relevar y describir los estudios que se han llevado a cabo en nuestra región en la temática mencionada.

\section{Método}

Se realizó una búsqueda en septiembre de 2015 en las bases de datos Pespsic, Scielo, Dialnet y Redalyc, bajo los descriptores "funciones ejecutivas" y "comprensión lectora" de forma combinada (AND).

Solo se incluyeron aquellos trabajos de investigación que indagaran las relaciones entre la comprensión de textos y los mecanismos de la inhibición y la actualización ejecutiva, llevados a cabo en la región iberoamericana. Se excluyeron aquellas investigaciones en donde se indagara la relación de las funciones ejecutivas y la lectura, es decir a nivel de la decodificación de la palabra. También se excluyeron aquellos trabajos que indagaran las relaciones entre comprensión de textos y funcionamiento ejecutivo en poblaciones que sufrieran alguna patología como TDAH, trastornos específicos del aprendizaje, del lenguaje o trastornos del espectro autista. 


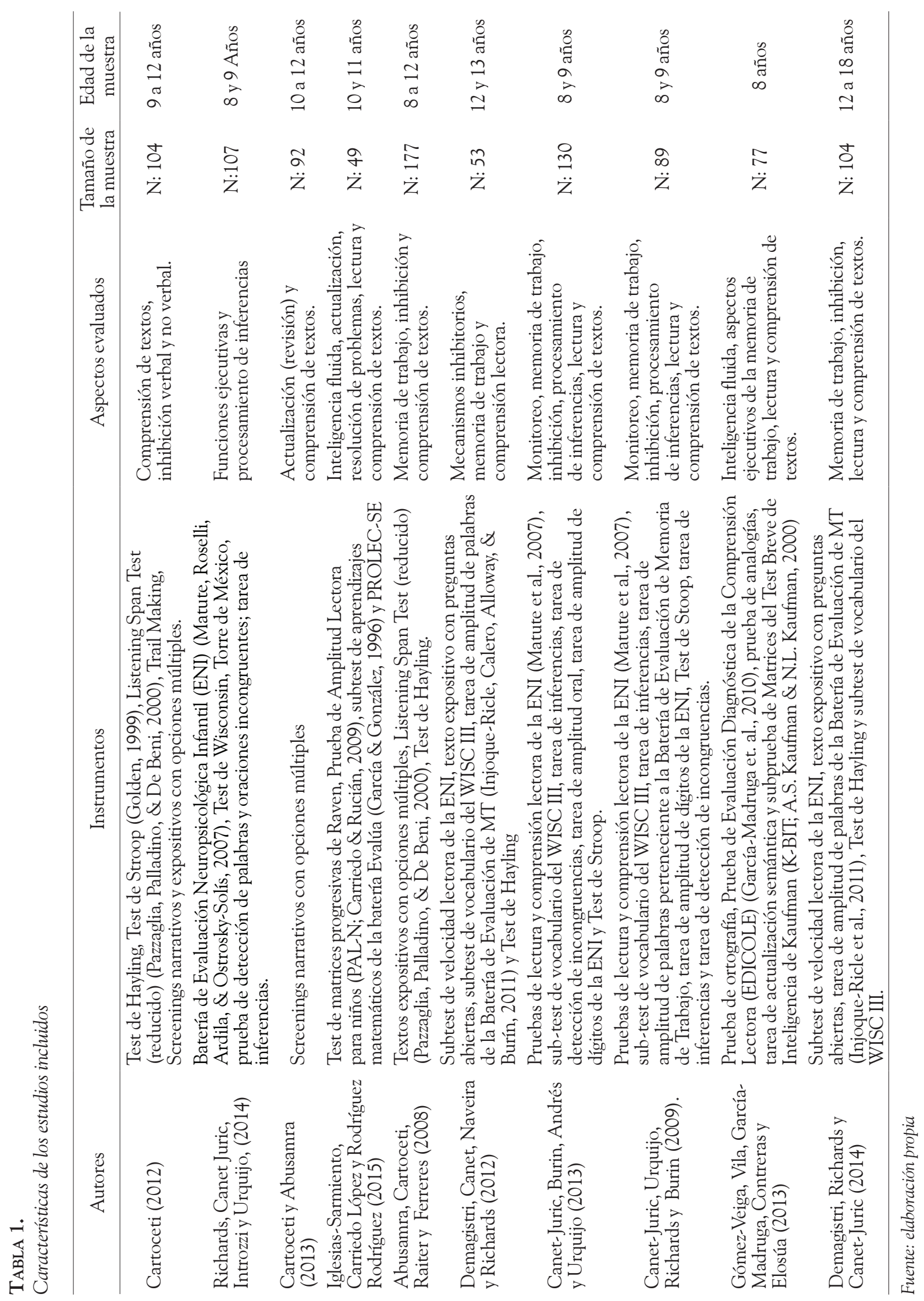




\section{Resultados}

La búsqueda arroja un resultado final de 10 investigaciones de campo, la cuales indagan las relaciones entre las funciones ejecutivas y la construcción del significado a partir del texto. Muchos de los trabajos indagan otras funciones ejecutivas más allá de la inhibición y de la actualización, pero la descripción que se realiza en este escrito se centrará únicamente en estas dos (ver Tabla 1).

\section{Actualización y comprensión lectora}

La función ejecutiva de actualización ha sido descrita en la literatura como un mecanismo relevante para la construcción del significado global y coherente, en la medida en que dicha tarea necesita de la integración de la información que propone el texto con la información almacenada en la memoria a largo plazo del lector. (Carreti et al., 2005).

Cartoceti y Abusamra (2013) examinaron el papel del mecanismo de actualización en la comprensión textual. En su estudio mostraron cómo los malos comprendedores tienen dificultades en la actualización del modelo de situación, es decir, los malos comprendedores tienen dificultades o bien para inhibir información o bien para mantener activa aquella que es relevante o que debe ser revisada en el momento de la lectura. Inhibición y actualización parecen actuar de forma mancomunada en el procesamiento textual.

Para este estudio, las investigadoras utilizaron dos textos que diseñaron de tal manera que el lector debía en un primer momento construir una representación del texto, y en un segundo momento construir otra representación que contradecía la primera parte del mismo.

Gómez-Veiga et al. (2013) indagaron las relaciones entre memoria operativa, inteligencia fluida y comprensión textual. La memoria operativa, constructo que nos interesa por sobre los otros indagados a los fines de esta revisión, fue evaluada por medio de una prueba de analogías y una prueba de actualización semántica. Los autores españoles demostraron que las tareas de memoria operativa son un buen predictor del nivel de comprensión lectora sobre todo, la prueba de actualización semántica que requiere seleccionar información relevante y suprimir la irrelevante.

La prueba de actualización semántica requiere que el niño recuerde una lista de palabras cada vez mayor, palabras que se refieren a objetos concreto que el niño conoce. El número de palabras que se presenta cada vez es mayor. A su vez, se le pide al niño que organice las palabras con un criterio disímil al orden en el que fueron presentadas, por ejemplo, en relación con el tamaño de los objetos a los que se refieren. La prueba supone que el sujeto deba elegir un criterio semántico, como el tamaño para ordenar las palabras. Esto supone por un lado, ir actualizando los conocimientos almacenados con un nuevo criterio, e inhibir información irrelevante para la tarea.

En el trabajo llevado a cabo por Iglesias-Sarmiento et al. (2015) se evidencia que el mecanismo de actualización es un buen predictor de las diferencias individuales en comprensión lectora. La relación entre actualización y comprensión lectora no parece estar mediatizada por procesos de dominio general como la inteligencia fluida, ni de dominio especifico como el procesamiento léxico.

El instrumento utilizado para evaluar la función ejecutiva de actualización fue la Prueba de Amplitud Lectora para niños (PAL-N; Carriedo \& Rucián, 2009) en la que se pide al sujeto que recuerde la última palabra de un conjunto de frases en un orden secuencial.

A grandes rasgos podemos observar que todos los trabajos afirman el rol que cumple el mecanismo de actualización en la comprensión eficaz de un texto. No obstante, los instrumentos con los que se evalúa dicha función ejecutiva son diferentes en cada caso, cuestión que dificulta afirmar que nos referimos al mismo proceso cognitivo que se pone en juego en la construcción del significado a partir del texto.

\section{Inhibición y comprensión lectora}

Además del mecanismo de actualización, la inhibición es otra de las funciones que ha sido ampliamente indagada en relación con la comprensión lectora. 
Para acceder al significado del texto es necesario inhibir información irrelevante o inapropiada que pueda incidir de forma negativa en la comprensión (De Beni et al., 1998).

En esta línea, Cartoceti (2012) ha evidenciado la relación estrecha que existe entre el control inhibitorio y la comprensión textual. Dicha autora evaluó a los participantes con una prueba que mide eficacia lectora, y con una prueba de comprensión, en la que se incluían cuatro screening desde el modelo teórico de multicomponencial (Abusamra, Ferres, et al., 2010). Los procesos inhibitorios se evaluaron a través del Test de Hayling y el Test de Stroop y la prueba de Trail Making (Test del trazo).

El Test de Hayling es una prueba que evalúa el procesamiento verbal y consta de dos partes, cada una con 15 oraciones a completar. En la primera parte del instrumento las oraciones se deben completar con una palabra coherente, y en la segunda parte, con palabras que no sean coherentes con la preposición. El Test de Stroop, también trabaja con material verbal y se trata de un instrumento formado por palabras con colores determinados, en dicho test se le pide al sujeto que inhiba la lectura de palabras (tarea automática), y nombre los colores de las mismas. El Test del Trazo, en la primera parte implica que el niño una números del 1 al 8 con un trazo, y en la segunda parte debe unir los números alternando los colores de los mismos.

En este trabajo, se halló que los comprendedores con dificultades tienen un déficit en los mecanismos de inhibición y supresión de la información, específicamente en el dominio verbal. Esta debilidad en la supresión de información irrelevante, no permite que el sujeto jerarquice la información y seleccione la relevante en el procesamiento textual.

En otro trabajo, Richards et al. (2014) indagaron las relaciones existentes entre las funciones ejecutivas y el procesamiento de inferencias. Las dimensiones evaluadas fueron: memoria de trabajo, planificación, monitoreo, y flexibilidad cognitiva.

La inhibición fue evaluada con el Test de Stroop que mencionamos anteriormente. Los autores encontraron escasas relaciones entre las habilidades de funcionamiento inhibitorio con ambos tipos de inferencias. Estos resultados parecen ser contradic- torios con los comunicados por Cartoceti (2012), no obstante, si bien el procesamiento de inferencias es un componente importante de la comprensión lectora, el mismo no agota dicho constructo.

Demagistri, et al. (2012) examinaron vocabulario, memoria de trabajo, inhibición, y fluidez lectora, separando a los sujetos según su bajo o alto rendimiento en comprensión lectora. El mecanismo de inhibición se evaluó con el Test de Hayling, y la comprensión lectora a través de la lectura silente de un texto y la respuesta a 10 preguntas abiertas sobre el mismo.

Los autores concluyen en que los déficits en memoria de trabajo e inhibición son característicos en sujetos con problemas de lectura. Además, se demostró que el mecanismo de inhibición está formado por al menos tres procesos: acceso, borrado y restricción. El acceso se refiere al control de la entrada de la información irrelevante al foco atencional, el borrado se ocupa de suprimir la información irrelevante durante el procesamiento de la información y la restricción se ocupa de inhibir las respuestas preponderantes, pero inapropiadas.

Por otro lado, Canet-Juric et al. (2013) se interesaron en indagar el perfil cognitivo de los malos comprendedores. Se evaluó el control inhibitorio por medio del Test de Stroop. La comprensión lectora fue evaluada con la pruebas de comprensión de lectura en voz alta y lectura silenciosa de la Batería de Evaluación Neuropsicológica Infantil.

En este estudio los autores plantean que los sujetos que tienen dificultades de comprensión no parecen tener dificultades con la inhibición de información irrelevante para la tarea. En otro trabajo, los mismos autores (Canet-Juric et al., 2009) utilizando los mismos instrumentos de medida plantean que el mecanismo de inhibición no parece ser un buen discriminador entre comprendedores con y sin dificultades. Esta afirmación vuelve a resultar contradictoria con las afirmaciones de Cartoceti (2012). Esto podría explicarse, porque los instrumentos y criterios que utilizan ambos trabajos para dividir a la población en buenos y malos comprendedores son disimiles.

En otro estudio llevado a cabo por Demagistri et al. (2014) evaluaron a dos grupos, uno, entre 12 
y13 años y otro, entre 17 y18 años. Indagaron las relaciones entre memoria de trabajo, inhibición y comprensión lectora. Esta última fue evaluada con dos textos diferentes en cada grupo sobre los que se formularon preguntas abiertas. La inhibición fue indagada con el Test de Hayling.

El trabajo muestra que los sujetos con rendimiento alto en comprensión lectora del primer grupo (12-13 años) muestran un desempeño superior en el control inhibitorio, mientras que en el segundo grupo (17-18 años) las diferencias no fueron significativas entre los buenos y los malos comprendedores, en el campo de la inhibición.

Finalmente, Abusamra et al. (2009) indagaron las relaciones entre la comprensión textual, la memoria de trabajo y el control inhibitorio. La comprensión fue evaluada a través de textos informativos y preguntas sobre los mismos que requerían respuestas literales e inferenciales. La inhibición fue evaluada con el Test de Hayling. En este estudio los autores muestran que los malos comprendedores parecen fallar en el sostenimiento de la información necesaria, el procesamiento y almacenamiento de la información relevante. Se vuelve a señalar en este trabajo la estrecha relación existente entre los mecanismos de inhibición y el exitoso desempeño a la hora de comprender un texto.

En contraposición con el caso de la actualización, el corpus no afirma en todos los casos que la función ejecutiva de inhibición se relaciona con la comprensión de textos. Las inconsistencias que aparecen entre las investigaciones podrían deberse a la diversidad con la que se evalúan los procesos cognitivos, ya sea el funcionamiento o la comprensión textual. Otra de las variables que parece incidir en las diferencias atribuidas a la inhibición en la comprensión de textos parece ser el desarrollo, cuestión que solo aparece en uno de los trabajos seleccionados.

\section{Discusión}

Este trabajo tuvo como objetivo seleccionar y describir investigaciones que mostraran la conexión entre las funciones ejecutivas de inhibición y actualización con la comprensión textual. En suma, se podría afirmar que existen relaciones entre los mecanismos de inhibición y actualización, y la construcción de una representación mental coherente del texto. No obstante, tanto la comprensión textual como las funciones ejecutivas son constructos complejos, de difícil definición y evaluación. Esto último se evidencia en la diversidad de instrumentos utilizados para medir cada uno de los constructos, que impide notablemente la comparación de los resultados de las investigaciones.

En el corpus descrito, por ejemplo, el control inhibitorio verbal ha sido analizado con dos pruebas: el Test de Stroop, el Test de Hayling. La pregunta que subyace es: ise refieren las investigaciones a la misma inhibición? Suponiendo que ambos tests miden constructos diferentes. Los resultados parecen mostrar que el proceso de inhibición se asocia en algunos casos con la comprensión lectora (Abusamra et al., 2009; Cartoceti, 2012; Demagistri et al., 2012) y en otros no (Canet-Juric, et al., 2013; Richards et al., 2104; Canet-Juric, et al., 2009). Además, las relaciones entre la comprensión textual y la inhibición parecen cambiar a lo largo del desarrollo (Demagistri et al., 2014). Creo que estos puntos deben ser indagados de forma más exhaustiva en futuras investigaciones.

En relación con el proceso de actualización se referencian tres instrumentos distintos: una prueba de actualización semántica, la Prueba de Amplitud Lectora, y un texto diseñado específicamente para evaluar la actualización mientras se lee. Nuevamente inos referimos al mismo proceso cognitivo en todos los casos? A diferencia de la inhibición, la actualización parece ser un buen predictor del desempeño en comprensión lectora en todos los casos descritos (Iglesias-Sarmiento et al., 2015; Gómez-Veiga et al., 2013; Cartoceti \& Abusamra, 2013).

Con respecto a la comprensión lectora aparece el mismo problema, en algunos casos dicha habilidad cultural es evaluada con un texto a resolver con preguntas abiertas, en otros, con textos a ser resueltos con preguntas de opción múltiple. Estas divergencias metodológicas impactan en los criterios a la hora de delimitar sujetos con buenos y malos desempeños, cuestión que oscurece nueva- 
mente la comparación de los resultados entre las investigaciones.

Se considera importante señalar para futuras investigaciones la construcción de pruebas o la adaptación de instrumentos que indaguen las funciones ejecutivas de actualización e inhibición en el campo especifico de la comprensión de textos. El instrumento diseñado por Cartoceti y Abusamra (2013) en el caso de la actualización es un buen ejemplo de ello.

La homogeneidad de los instrumentos hará posible la comparación de los resultados y posibilitará afirmar de forma más rigurosa qué procesos cognitivos están asociados o no con la comprensión de textos.

Como Cahin y Oakhill (2006) considero que identificar y estudiar los diferentes procesos que permiten la comprensión del texto hará posible diagnosticar rendimientos pobres e intervenir sobre ellos. Así mismo, las relaciones entre funciones ejecutivas y desempeño textual serían un nodo importante a la hora de examinar a quienes presentan dificultades en comprensión, como ya lo plantean algunas investigaciones (Elosúa, GarcíaMadruga, Vila, Gómez-Veiga, \& Gil, 2013), pero aún quedan muchos interrogantes sobre dichas relaciones.

\section{Referencias}

Abusamra, V., Cartoceti, R., Raiter, A., \& Ferreres, A. (2008). Una perspectiva cognitiva en el estudio de la comprensión de textos. Psico, 39, 352-361.

Abusamra, V. Casajús, A. Ferreres, A. Ratier, A. De Beni, R., \& Cornoldi, C. (2011). Programa Leer para Comprender. Libro Teórico. Buenos Aires: Paidos.

Abusamra, V., Ferreres, A., Raiter, A., De Beni, R. \& Cornoldi, C. (Comps.) (2010). Test Leer para Comprender. Buenos Aires: Paidós.

Canet-Juric, L., Burin, D., Andrés, M. L., \& Urquijo, S. (2013).Perfil cognitivo de niños con rendimientos bajos en comprensión lectora. Anales de Psicología, 29(3), 996-1005.

Canet-Juric, L., Urquijo, S., Richards, M. M., \& Burin, D. (2009). Predictores cognitivos de niveles de comprensión lectora mediante análisis discrimi- nante. International Journal of Psychological Research, 2(2), 99-111.

Carretti, B., Borella, E., Cornoldi, C., \& De Beni, R. (2009). Role of working memory in explaining the performance of individuals with specific reading comprehension difficulties: A meta-analysis. Learning and Individual Differences, 19(2), 246-251.

Carretti, B., Cornoldi, C., De Beni, R., \& Romanò, M. (2005). Updating in working memory: A comparison of poor and good comprehenders. Journal of Experimental Child Psychology, 91(1), 45-66.

Carriedo, N., \& Rucián, M. (2009). Adaptación para niños de la prueba de amplitud lectora de Daneman y Carpenter (PAL-N) [Adaptation for children of the Reading Span Test of Daneman \& Carpenter (PAL-N)]. Infancia E Aprendizaje, 32(3), 449-465.

Cartoceti, R. \& Abusamra, V. (2013). El rol del mecanismo de actualización en la comprensión de textos. Revista Neuropsicología Latinoamericana, 5(2), 1-10.

Cartoceti, R. (2012). Control inhibitorio y comprensión de textos: evidencias de dominio especifico verbal. Revista Neuropsicología Latinoamericana, 4(1), 65-85.

Clair-Thompson, H. L., \& Gathercole, S. E. (2006). Executive functions and achievements in school: Shifting, updating, inhibition, and working memory. The quarterly journal of experimental psycho$\log y$, 59(4), 745-759.

De Beni, R., Cornoldi, C., Carretti, B., \& Meneghetti, B. (2003). Unova Guida alla Comprensione del testo. Trento: Erickson.

De Beni, R.; Palladino, P.; Pazzaglia, F., \& Cornoldi, C. (1998). Increases in intrusion errors and working memory deficit of poor comprehenders. Quarterly Journal of Experimental Psychology, 51, 305-320.

Demagistri, M. S., Richards, M., Canet, L (2014) Incidence of Executive Functions on Reading Comprehension Perfomance in Adolescents. Electronic Journal of Research in Educational Psychology, 12(2), 343-370.

Demagistri, M. S., Canet, L., Naveira, L., \& Richards, M. (2012) Memoria de trabajo, mecanismos inhibitorios y rendimiento lecto-comprensivo en grupos de comprendedores de secundaria básica. Revista Chilena de Neuropsicología, 7(2), 72-78. 
Elosúa, M.R., García- Madruga, J.A., Vila, J.O., GómezVeiga, I., \& Gil, L. (2013). Improving reading comprehension: From metacognitive intervention on strategies to the intervention on working memory executive processes. Universitas Psychologica, 12(5), 1425-1438.

García, J., \& González, D. (1996). Batería Psicopedagógica Evalúa. Madrid: Editorial EOS.

García-Madruga, J. A., Gutiérrez Martínez, F., \& Vila Chavez, J. O. (2012). El desarrollo de la Memoria. En José A. Castorina; Mario Carretero (Comps.), Desarrollo Cognitivo y Educación [II]. Los inicios del conocimiento (pp. 47-66) Buenos Aires, Paidós.

García Madruga, J. A., Pérez, E., Gómez-Veiga, I., Orjales, I., Gil, L., Elosúa, R., \& López-Escribano, C. (2010). Prueba de comprensión lectora para enseñanza primaria: EDICOLE (evaluación diagnóstica de la comprensión lectora). No publicado.

Golden, C. J. (1999). Stroop. Test de Colores y Palabras. Manual de aplicación. Madrid: TEA Ediciones.

Gómez-Veiga, I., Vila, J. O., García-Madruga, J. A., Contreras, A., \& Elosúa, M. J. (2013). Comprensión lectora y procesos ejecutivos de la memoria operativa. Psicología Educativa, 19, 103-111.

Iglesias-Sarmiento, V., Carriedo López, N., \& Rodríguez Rodríguez, J. L. (2015). Updating executive function and performance in reading comprehension and problem solving. Anales de Psicología, 31(1), 298-309.

Injoque-Ricle, I., Calero, A. D., Alloway, T. P., \& Burin, D.I. (2011). Assessing working memory in Spanishspeaking children: Automated Working Memory Assessment Battery adaptation. Learning and Individual Differences, 21, 78-84.

Kaufman, A. S., \& Kaufman, N. L. (2000). K-BIT, test breve de inteligencia de Kaufman [KBIT, the brief test of intelligence by Kaufman]. New York: PsychCorp.

Matute, E., Roselli, M., Ardila, A., \& Ostrovsky-Solís, F. (2007). Evaluación Neuropsicológica Infantil (ENI). Manual de aplicación. México D.F., México: El Manual Moderno.

Miyake, A., Friedman, N. P., Emerson, M. J., Witzki, A. H., \& Howerter, A. (2000). The unity and diversity of executive functions and their contributions to complex "frontal lobe" tasks: A latent variable analysis. Cognitive Psychology, 41(1), 49-100.

Musso, M. F. (2009). Funciones ejecutivas y control ejecutivo: una revisión bibliográfica mirando la arquitectura de la mente, Revista de Psicología, 5(9), 107-123.

Pazzaglia, F., Palladino, P., \& De Beni, R. (2000). Presentazione di uno strumento per la valutazione della memoria di lavoro verbale e sua relazione con i disturbi della comprensione. Psicología Clinica dell Sviluppo, 3, 465-486.

Piacente, T. (2009). Especificidad de la evaluación psicológica en los procesos de aprendizaje del lenguaje escrito. Evolución y estado actual. RIDEP, 28(2), 135-148.

Piacente, T. (2012). Alfabetización inicial y alfabetización académica. Investigación y Evaluación de los procesos implicados en el aprendizaje del lenguaje escrito, RIDEP, 33(1), 9-30.

Richard's, M., Canet Juric, M. L., Introzzi, I., \& Urquijo, S. (2014). Intervención diferencial de las funciones ejecutivas en inferencias elaborativas y puente. Avances en Psicología Latinoamericana, 32(1), 5-20.

Stelzer, F \& Cervigni, M. A. (2011). Desempeño académico y funciones ejecutivas en infancia y adolescencia. Una revisión de la literatura. Revista de Investigación en Educación, 9(1), 148-156.

Tirapu-Ustárroz, J., García-Molina, A., Luna-Lario, P., Roig-Rovira T., \& Pelegrín-Valero, C. (2008a). Modelos de funciones y control ejecutivo (I) Revista de Neurología, 46(11), 684-669.

Tirapu-Ustárroz, J., García-Molina, A., Luna-Lario, P., Roig-Rovira T., \& Pelegrín-Valero, C. (2008b). Modelos de funciones y control ejecutivo (II) Revista de Neurología, 46(12), 742-750.

Tirapu-Ustárroz, J., Muñoz-Céspedes, J. M., \& PelegrínValero, C. (2002). Funciones ejecutivas: necesidad de una integración conceptual. Revista de Neurología, 34, 673-685.

Zabaleta, V. (2009). La competencia lectora en los programas nacionales e internacionales de evaluación educativa. Orientación y Sociedad. Revista Internacional e Interdisciplinaria de Orientación Vocacional Ocupacional, 8-9, 109-129. 
